\title{
Severe Factor XII Deficiency with Unusual Presentation during Pregnancy: A Case Report
}

\section{Salwa Bakr ${ }^{1,2^{*}}$}

${ }^{1}$ Department of Clinical Pathology/Hematology, College of Medicine, Fayoum University, Egypt

2Imam Abdulrahman Bin Faisal University, Dammam, Saudi Arabia

"Corresponding author: Salwa Bakr, Department of Clinical Pathology/ Hematology, College of Medicine, Fayoum University, Egypt, Tel: + 966541442981; E-mail: Sbm00@fayoum.edu.eg

Received date: July 02, 2018; Accepted date: July 28, 2018; Published Date: August 06, 2018

Copyright: $\odot 2018$ Bakr S. This is an open-access article distributed under the terms of the Creative Commons Attribution License, which permits unrestricted use, distribution, and reproduction in any medium, provided the original author and source are credited.

\begin{abstract}
Factor XII deficiency has been reported to be associated with recurrent miscarriages, however published studies seem contradictory and need to be elucidated. Here, we report a rare case of severe FXII deficiency with unusual presentation and unexpected outcomes during pregnancy. A 26-year-old multiparous female with prolonged APTT that was discovered during her routine gestational checkup. Coagulation profile showed extremely low FXII activity $(2 \%)$. PC, PS, ATIII, LAC and $\mathrm{aCL}$ antibodies were unremarkable. She had three full term normal pregnancies without complications with normal vaginal delivery of normal healthy infants with no history of any previous pregnancy loss. Although she opted to take oral contraceptive pills with her Ob-Gyn, no thromboembolic episode or any other complications have been reported. In conclusion, the possibility of normal pregnancy with FXII deficiency merits to be documented so a case series can be generated. We affirm that the possible association of pregnancy loss with FXII deficiency merits reevaluation.
\end{abstract}

Keywords: Hageman factor; Factor XII deficiency, Pregnancy loss; Thrombosis, Recurrent miscarriage

\section{Introduction}

Factor XII (Hageman factor) is a plasma serine protease known to be implied in triggering the intrinsic pathway of blood coagulation and fibrinolysis cascade [1].

Factor XII deficiency is a rare inherited autosomal recessive, blood disorder that causes persistent prolongations of blood clotting in vitro test (Activated Partial Thromboplastin Time), but whose deficiency do not have clinical bleeding tendencies [2].

FXII deficiency is reported to be associated with thromboembolic disorders, recurrent miscarriages and placental abruption $[3,4]$. Although the pathophysiology of its relationship with overt thrombosis is still unclear [5], it has been argued that FXIIa plays a double role in coagulation cascade and its deficiency could cause miscarriage by inducing formation of microthrombi [6].

On the contrary, Matsuura et al. suggested that deficiency of FXII is not certainly associated with pregnancy loss even for patients with severe deficiency [3]. For more perplexities, in a small series of cases with FXII deficiency mild postpartum hemorrhage have been reported $[1,7]$.

As previous published studies showed controversy, still it has been unclear whether factor XII deficiency causes any disorder during pregnancy [1]. Here we report a case of severe FXII deficiency in a multiparous female, who has normal uneventful two pregnancy courses, hence a case series can be generated.

\section{Case Report}

A 24-year-old multiparous female was scheduled in outpatient clinic at King Khalid University Hospital for routine postnatal follow up of her second pregnancy. She has been diagnosed with severe FXII deficiency (2\%) during her routine prenatal checkup of her second gestation. Her two courses of gestation and delivery were normal. The courses of her two pregnancies were uneventful till the end with normal vaginal deliveries. She was asymptomatic and clinically well, with no history of heavy vaginal bleeding during perperium. She had not experienced any past history of any abnormal spontaneous bleeding episodes or evidence of thrombosis. She did not have any history of previous medication or heparin therapy. No history of previous pregnancy loss. There is no known family history of bleeding or thrombotic tendencies.

The general physical examination was normal, within average built with normal vital signs without sign of jaundice, pallor, cyanosis or bruise marks. Laboratory investigation showed normal complete blood picture (Hb: $12 \mathrm{gm} / \mathrm{dl}$, platelet count: $380 \times 10 / \mathrm{L})$. Coagulation profile showed PT=15.7 (Normal reference $=11.5-16.5)$, APTT $<120 /$ sec. even on repeated APTT test (Normal reference $=26-39$ ) and $\mathrm{TT}=16.5$ (Normal reference $=14-21$ ). Mixing studies showed complete correction of APTT, suggesting factor deficiency. Further coagulation testing showed extremely low FXII activity (2\%, reference value $70 \%-145 \%)$ with elevation of levels of coagulation factors (XI, IX, vWF Ag and fibrinogen). Protein $\mathrm{C}$, protein S, antithrombin III, Lupus anticoagulant and anticardiolipin antibodies $\operatorname{IgG}$ and $\operatorname{IgM}$ were ordered to rule out possible potential prothrombotic causes. All the results of these were unremarkable (Table 1). 
Citation: Bakr S (2018) Severe Factor XII Deficiency with Unusual Presentation during Pregnancy: A Case Report. Med Rep Case Stud 3: 161.

Page 2 of 4

\begin{tabular}{|c|c|c|}
\hline Parameters & Patient's Result & Normal range \\
\hline \multicolumn{3}{|c|}{ Coagulation Screening profile } \\
\hline PT & 15.7 & $11.5-16.5 \mathrm{~s}$ \\
\hline APTT & $>120$ & $26-39 s$ \\
\hline TT & 17.5 & $14-21 \mathrm{~s}$ \\
\hline \multicolumn{3}{|l|}{ Coagulation Factors Assay } \\
\hline FXII & $2 \%$ & $70 \%-145 \%$ \\
\hline $\mathrm{FXI}$ & $200 \%$ & $50 \%-150 \%$ \\
\hline FVIII & $120 \%$ & $50 \%-200 \%$ \\
\hline FIX & $200 \%$ & $50 \%-150 \%$ \\
\hline Fibrinogen & 4.80 & $2.0-4.0 \mathrm{~g} / \mathrm{L}$ \\
\hline \multicolumn{3}{|l|}{ Hypercoagulability Profile } \\
\hline PC & $139 \%$ & $70 \%-140 \%$ \\
\hline PS & $80 \%$ & $65 \%-140 \%$ \\
\hline ATIII & $105 \%$ & $80 \%-120 \%$ \\
\hline \multicolumn{3}{|l|}{ Immunological Assay } \\
\hline Anticardiolipin IgG & Negative & $<20 \mathrm{GPL} \mathrm{U} / \mathrm{mL}$ \\
\hline Anticardiolipin IgM & Negative & $<10 \mathrm{MPL} \mathrm{U} / \mathrm{mL}$ \\
\hline$\beta 2$-GPI antibodies & Negative & $<18 \mathrm{U} / \mathrm{mL}$ \\
\hline \multicolumn{3}{|l|}{ Lupus Anticoagulant } \\
\hline DRVVT ratio & 0.60 & $<1.20$ \\
\hline Kaolin clotting time ratio & 0.80 & $<1.10$ \\
\hline
\end{tabular}

Table 1: Laboratory report for coagulation parameters; (PT: prothrombin time; APPT: activated partial thromboplastin time; TT: thrombin time; PC: protein C; PS: Protein S; ATIII: antithrombin III; DRVVT: diluted Russell's viper venom time; Ig: immunoglobulin; $\beta 2-G P I, \beta 2-$ glycoprotein 1$)$. The cut off values for anti-cardiolipin (aCL) and anti- $\beta 2$-glycoprotein 1 (a $\beta 2 \mathrm{GP} 1)$ were established following the International Consensus Guidelines on Anticardiolipin and Anti-B2-Glycoprotein I testing with the $99^{\text {th }}$ percentile of the healthy blood donor.

Patient was categorized during her second gestational period according to hospital venous thromboembolic risk assessment tool as moderate risk category (score: 2).The course of her two pregnancies were uneventful till the end with normal vaginal deliveries. She gives in all her deliveries spontaneous birth to healthy babies within average weight and good Apgar score, without neither cord stump bleeding nor skin bruises. Post-delivery, she had uneventful smooth recovery with normal lochial discharge.

At three months' follow up, she opted to take oral contraceptive pills (OCP), after discussing the available contraceptive options with her doctor. At six months' follow up, OCP's were well tolerated with no thromboembolic episode or any other complication. The patient reports that the child is doing well. Informed consent was taken from the patient for the publication of this case report.

\section{Discussion}

Factor XII deficiency theoretically, may carry either bleeding risk as a result of reduced coagulation factors or thrombosis risk as a consequence of reduced fibrinolytic activity [8].

In fact, pregnancy is associated with physiological changes result in hypercoagulability and hypofibrinolytic state with a consequence of increase the risk of thromboembolism and recurrent pregnancy loss [3]. Although it was suggested that microthrombi formation might be the reason behind the reported cases of miscarriage with FXII deficiency [5], its relationship with overt venous thrombosis is still unclear $[6,9]$. Here we report an unexpected outcome of a multiparous female with severe FXII deficiency, who had uneventful two pregnancy without any complications, and normal healthy infants were delivered.

The clinical implications of FXII deficiency are controversial. Several studies have described identified FXII deficiency as a risk factor for recurrent miscarriages, others failed to find such an association [10]. Recurrent pregnancy loss has been reported in cases 
Page 3 of 4

with decreased FXII activity $[1,4,9]$. Some studies found an association between the presence FXII autoantibodies with recurrent pregnancy loss [1,3-5]. Hence, Matsuura et al. assumed that this coexisted antibodies might be the cause of recurrent miscarriage [3].

In another study, Matsubayashi et al. proved that decreased activity of F-XII was found to be associated with recurrent in vitro fertilization failure. Infertility was one of the complication that has been reported with the hypercoagulability in a light deficiency of the factor XII. [11]. Many studies suggested the importance of using low dose of aspirin (125 mg/day) and preconception folic acid ( $5 \mathrm{mg} /$ day) till conception achieved. Follow up is essential in such ladies from the week 12 of their pregnancy with antithrombotic prophylaxis with low molecular weight heparin to avoid loss of pregnancy [5].

Since coagulation factor XII first discovery in 1955, its deficiency was, totally surprisingly, connected with thrombosis instead of bleeding. This reported association originated after John Hageman, the index patient with this defect, had died of pulmonary embolism [12]. Nevertheless, it is often overlooked that this occasion followed a traumatic pelvic fracture with consequent prolonged immobilization. Hence several reports have highlighted the correlation between FXII deficiency and thrombosis [8]; however, a causal relationship is still questioned $[6,13]$.

In contradictory, some researchers rejected the certainty of considering FXII (Hageman factor) deficiency as a prothrombotic condition since there many well-known associated risk factors for thrombosis; such as pregnancy, postpartum period, surgery, smoking, hypertension, diabetes mellitus, dyslipidemia for arterial thrombosis, and trauma for venous thrombosis $[6,7,14]$. Lao et al. and Matsuura et al. suggested that normal pregnancy is a possible outcome in cases with congenital FXII deficiency and its association with recurrent miscarriage need to be reevaluated $[3,15]$.

Recently, some researchers found that mice lacking FXII has thrombo-protective effect [16]. Renne et al. showed that inhibition of factor XII is a novel safe therapeutic anticoagulant strategy that targets thrombosis without the side effect of increase bleeding [17]. In another study, it was pointed out that deficiency of Hageman factor neither causes nor protects from venous thrombosis [12]. Moreover, Girolami et al. concluded that even patients with severe homozygous deficiency of FXII are neither at risk of bleeding nor thrombosis even after major surgical procedures [18]. Furthermore, some authors found that including FXII assay in the battery of prothrombotic tests is an unjustified claim [6].

For more perplexities, while there is a common agreement that FXII deficiency usually does not manifest in a bleeding tendencies except for few exceptional reported cases $[2,3,7,19,20]$. Girolami et al. reported that Occasional mild bleeding that was recorded in pregnancy with FXII deficiency was due to an associated cause as thrombocytopenia, $3^{\text {rd }}$ degree perineal tear or decrease uterine contraction [7].

Finally, the impact of FXII deficiency on pregnancy outcome is still ambiguous and debatable [3]. Published studies seem contradictory and need to be elucidated. Both recurrent miscarriage, mild postpartum hemorrhage and normal pregnancy have been found in a small series with factor XII deficiency [7]. Here we reported, to the best of our knowledge, the second case in the literature of a multiparous woman with severe FXII deficiency $(2 \%)$ who was progressed in her pregnancies to term without any complications neither thrombotic nor spontaneous bleeding tendencies. Hence, we do think that the possible relationship of FXII deficiency with normal pregnancy merits to be documented so a case series can be generated.

\section{Conclusion}

Our study highlights the possibility of normal pregnancy and uncertainty of miscarriage with severe FXII deficiency. We assert that the possible association of recurrent pregnancy loss with FXII deficiency merits reevaluation.

\section{Disclosure Statement}

The author has no conflict of interest

\section{Acknowledgment}

I am grateful to Dr. Ghada ElGohary, Consultant of Adult Hematology/BMT, King Khalid University Hospital, Riyadh, Saudi Arabia; Ain Shams University Hospital, Cairo, Egypt for her valuable time and support.

\section{References}

1. Mariano M, Yadira P, Ana A, Manuel L, Jesús L (2011) Coagulation Factor XII Congenital Deficiency in Women with Recurrent Miscarriage. Int J Clin Med 2: 469-472.

2. Schindhelm RK, Wondergem MJ, Admiraal J, Nap G, ten Boekel E, et al. (2012) A Patient with a Prolonged Activated Partial Thromboplastin Time and a Deep Intracerebral Haemorrhage. Case Rep Neurol 4: 131-136.

3. Matsuura T, Kobayashi T, Asahina T, Kanayama N, Terao T (2001) Is factor XII deficiency related to recurrent miscarriage? Semin Thromb Hemos; 27: 115-120.

4. Pauer HU, Burfeind P, Köstering H, Emons G, Hinney B (2003) Factor $\mathrm{XII}$ deficiency is strongly associated with primary recurrent abortions. Fertil Steril 80: 590-594.

5. Girolami A, Morello M, Girolami B, Lombardi AM, Bertolo C (2005) Myocardial infarction and arterial thrombosis in severe (homozygous) FXII deficiency: no apparent causative relation. Clin Appl Thromb Hemost 11: 49-53.

6. Ogasawara MS, Aoki K, Katano K, Ozaki Y, Suzumori K (2001) Factor XII but not protein C, protein S, antithrombin III, or factor XIII is a predictor of recurrent miscarriage. Fertil Steril 75: 916-919.

7. Girolami A, Zocca N, Girolami B, Lombardi AM, Fabris F (2004) Pregnancies and oral contraceptive therapy in severe (homozygons) FXII deficiency: a study in 12 patients and review of the literature. J Thromb Thrombolysis 18: 209-212.

8. Lessiani G, Falco A, Nicolucci E, Rolandi G, Davi G (2009) Deep vein thrombosis and previous myocardial infarction in mild factor XII deficiency: a risk factor for both venous and thrombosis. J Thromb Thrombolysis 27: 348-351.

9. Sotiriadis A, Makrigiannakis A, Stefos T, Paraskevaidis E, Kalantaridou SN (2007) Fibrinolytic defects and recurrent miscarriage: a systematic review and meta-analysis. Obstet Gynecol 109: 1146-1155.

10. Sugi $T$ (2013) Kininogen-dependent antiphosphatidylethanolamine antibodies and autoantibodies to factor XII in patients with recurrent pregnancy losses. J Obstet Gynaecol Res 39: 1223-1229.

11. Matsubayashi H, Sugi T, Suzuki T, Uchida N, Atsumi H, et al. (2008) Decreased Factor XII Activity is Associated with Recurrent IVF-ET Failure. Am J Reprod Immunol 59: 316-322.

12. Girolami A, Vettore S, Lombardi AM (2007) About the significance or the insignificance of the factor XII C46T polymorphism: arebuttal. J Thromb Haemost 5: 2162-2163. 
Citation: Bakr S (2018) Severe Factor XII Deficiency with Unusual Presentation during Pregnancy: A Case Report. Med Rep Case Stud 3: 161. doi:10.4172/2572-5130.1000161

Page 4 of 4

13. Koster T, Rosendaal FR, Briet E, Vandenbroucke JP (1994) John Hageman's factor and deep-vein thrombosis: Leiden Thrombophilia Study. British Journal of Haematology 87: 422-424.

14. Girolami A, Candeo N, De Marinis GB, Bonamigo E, Girolami B (2011) Comparative incidence of thrombosis in reported cases of deficiencies of factors of the contact phase of blood coagulation. J Thromb Thrombolysis 31: 57-63.

15. Lao TT, Lewinsky RM, Ohlsson A, Cohen H (1991) Factor XII deficiency and pregnancy. Obstet Gynecol 78: 491-493.

16. Pham M, Stoll G, Nieswandt B, Bendszus M, Kleinschnitz C (2012) Blood coagulation factor XII-a neglected player in stroke pathophysiology. J Mo Med (Berl) 90: 119-126.
17. Renné T, Schmaier AH, Nickel KF, Blombäck M, Maas C (2012) In vivo roles of factor XII. Blood 120: 4296-4303.

18. Girolami A, Ruzzon E, Lombardi AM, Cabrio L, Randi ML (2004) Thrombosis-free surgical procedures in severe (homozygote) factor XII deficiency: report of four additional cases and literature review. Clin Appl Thromb Hemost 10: 351-355.

19. Didisheim P (1962) Hageman factor deflciency (Hageman Trait): Case report and review of the literature. Arch Intern Med 110; 74-81.

20. Ikkala E, Myllyla G, Nevanlmna HR (1971) Rare congenital coagulation factor defects in Finland. Scand J Haemat 8: 210-215. 